\title{
Evaluación de la Técnica del Drenaje Vesical Suprapúbico con Sonda de Folley comparada con el Drenaje Vesical Transuretral con el mismo tipo de sonda en las Histerectomías Vaginales
}

\author{
DEPARTAMENTO DE OBSTETRICIA Y GINECOLOGIA \\ FACULTAD DE MEDICINA - UNIVERSIDAD DE ANTIOQUIA \\ Fundación Hospitalaria San Vicente de Paúl \\ Asociación Antioqueña de Obstetricia y Ginecología \\ CENTRO DE INVESTIGACIONES MEDICAS \\ Dres.: Jaime Garcia Martínez*, Gabriel Uribe Espitia**, \\ Gabriel Tobón Londoño***, José León Carrero****, \\ Domingo Manotas Ramírez*****, Lilliana Alvarez******.
}

* Profesor Asociado Departamento de Obstetricia y Ginecología de la Facultad de Medicina de la Universidad de Antioquia.

* Jefe Departamento de Obstetricia y Ginecologia de la Facultad de Medici. na de la Universidad de Antioquia.

** Coordinador Grupo de Ginecología del Departamento de Obstetricia y Ginecologia de la Facultad de Medicina de la Universidad de Antioquia.
**** Residente de Tercer año de Obstetricia y Ginecologia de la Facultad de Medicina de la Universidad de Antioquia.

***** Residente de Segundo año de Obstetricia y Ginecologia de la Facultad de Medicina de la Universidad de Antioquia.

****** Bacterióloga, Profesora Asociada del Departamento de Bacteriología de la Facultad de Medicina de la Universidad de Antioquia. 


\section{INTRODUCCION}

Preocupados por la alta incidencia de infecciones urinarias postoperatorias después de la histerectomía vaginal y especialmente cuando van acompañadas de plastia anterior, realizamos el siguiente trabajo, comparando los resultados obtenidos de las pacientes en las que utilizábamos el método tradicional de drenaje vesical con sonda transuretral, con otro grupo de pacientes en las que utilizamos el drenaje con la misma sonda de Folley pero utilizando la técnica de la inserción suprapúbica descrita por Clif. ford R. Wheeles en su "Atlas de Cirugía Pelviana" edición de 1981.

Ya desde 1959 Kass y Sossen habian demostrado la presencia de bacteriurias de más de 100.000 colonias en el $98 \%$ de las pacientes a quienes se les debaja el cateter de drenaje vesical por más de cuatro días y estas cifras no se han modificado notoriamente en ningún centro ginecológico a pesar de las distintas técnicas de asepsia que se utilizaron y ni siquiera con la administración de antibióticos profilácticos. Lo que si ha hecho disminuir en forma muy amplia estas bacteriurias es el drenaje urinario por vía suprapúbica y en los últimos años se han diseñado varios equipos, algunos muy sofisticados y costosos que no están al alcance de nuestros hospitales de por sí escasos en recursos económicos.

Escogimos este tipo de cistostomía porque la sonda de Folley común, una vez insertada de la manera como la describiremos más adelante, no se suele salir de la vejiga durante el sueño ni con la actividad de la paciente, es bien tolerada, barata y la tienen todos los hospitales.

Tradicionalmente hemos utilizado en el Departamento de Obstetricia y Ginecología del Hospital Universitario San Vicente de Paúl de Medellín, la sonda vesical a permanencia por vía uretral para proteger y evaluar el funcionamien- to vesical, medido indirectamente por la presencia de residuo de orina menor de 100 cc luego de 72 horas de postoperatorio en la histerectomía vaginal. La sonda se deja abierta 48 horas. En las 24 horas siguientes se estimula el automatismo del músculo detrusor cerrando la sonda y abriéndola durante diez minutos cada tres horas. Finalmente se retira la sonda y luego de dos micciones espontáneas se mide el residuo. Cuando el residuo es mayor de $100 \mathrm{cc}$ se aplica nuevamente la sonda por un período de 48 horas.

\section{Objetivo propuesto}

Los objetivos propuestos en este trabajo fueron los siguientes:

1. Evaluar la técnica de drenaje vesical utilizada tradicionalmente en nuestro Departamento.

2. Evaluar una nueva técnica de drenaje vesical por vía suprapúbica teniendo en cuenta:

\subsection{Funcionamiento apropiado}

2.2. Tiempo de permanencia de la sonda

2.3. Valoración del residuo urinario

2.4. Frecuencia de bacteriurias postoperatorias

2.5 Complicaciones quirúrgicas de la técnica.

3. Comparar los resultados de las dos técnicas y sugerir recomendaciones.

\section{MATERIAL Y METODOS}

El trabajo realizado fue de tipo prospectivo y por el método "doble ciego". Se utilizaron 91 pacientes a quienes se les practicó histerectomía vaginal y plastias por prolapsos genitales en el período de enero de 1982 a mayo de 1983 (17 meses). 
A 38 pacientes se les practicó el drenaje convencional transuretral y a 53 el drenaje suprapúbico con la técnica que luego describiremos. A todas ellas se les tomó muestra de orina para el primer estudio bacteriológico pero para los resultados finales hubo necesidad de retirar 24 pacientes a quienes no se les pudo practicar segundo examen bacteriológico por diferentes causas.

\section{METODOLOGIA}

1. El grupo quirúrgico aplicó la técnica de drenaje vesical (transuretral o suprapúbico) de acuerdo con un estricto orden.

2. A todas las pacientes se les realizó el mismo tipo de preparación aséptica de ios genitales antes de la introducción de la sonda.

3. Se tomaron muestras de orina para, cultivo al instante de aplicar la sonda y en el momento de retirarla.

4. Se utilizó sonda de Folley No. 14 y equipos de drenaje cerrados.

5. Se consignaron las dificultades técnicas para cada uno de los dos métodos utilizados y sus complicaciones.

6. Se evaluó el tiempo de permanencia de la sonda y se analizó el residuo vesical.

7. Se consignó el tiempo de hospitalización y las causas que motivaron la mayor estancia.

8. Se analizaron los resultados de los cultivos de orina.

\section{Técnica de Cistostomia}

Nosotros utilizamos una variación de la técnica descrita en 1970 por los doctores Shute $\vee$ Mackinnon muy parecida a la publicada por el doctor Clifford R. Wheeless en 1981.
El procedimiento se realiza en el momento mismo en que se va a iniciar la intervención para la cual ha sido programada la paciente. Posición de litotomía, anestesia adecuada, correcto lavado de región suprapúbica $y$ de los genitales externos.

1. Se pasa pinza de Pean larga a través de la uretra hasta cara anterior de vejiga.

2. Se hace presión con la punta de la pinza cerrada hasta que sea fácilmente palpable por la pared anterior del hipogastrio, unos dos centímetros por encima del borde superior del pubis.

3. Se practica una pequeña incisión en la piel que se profundiza hasta vejiga para extraer a través de ella la punta de la pinza la cual a su vez se abre muy levemente para coger con firmeza la sonda de Folley.

4. Se saca de la vejiga la pinza de Pean con la sonda agarrada. Esto para tener la seguridad de que la sonda si quedara bien colocada dentro de la cavidad vesical. Se suelta la sonda y se infla el balón.

5. Se fija la sonda con un punto a la pared abdominal y su extremo distal se conecta a un equipo de drenaje cerrado.

La sonda se deja funcionando abierta por 48 horas. Después de este tiempo se inician los ejercicios vesicales abriendo y cerrando la sonda cada cuatro horas. Como la uretra queda libre, la micción espontánea puede iniciarse en cualquier momento razón por la cual la sonda se puede retirar sin necesidad de esperar el plazo convencional de 24 horas.

\section{RESULTADOS Y COMENTARIOS}

Funcionamiento apropiado de la sonda y complicaciones quirúrgicas de la técnica: 
En el grupo de 53 pacientes con cistostomía tuvimos dos perforaciones vesicales a cavidad abdominal atribuibles a mala técnica de inserción del cateter. En otras dos pacientes la sonda aparentemente bien colocada no funcionó y hubo necesidad de retirarlas. En las restantes 49 pacientes la sonda funcionó ,perfectamente bien y no hubo ningún tipo de complicación. Creemos que éstas complicaciones $(7.5 \%$ en nuestro trabajo) son totalmente evitables con un poco de experiencia. Las 38 pacientes con drenaje convencional no tuvieron ningún contratiempo.

Para los análisis siguientes se tuvieron en cuenta un total de 37 pacientes con drenaje suprapúbico y 30 pacientes con drenaje transuretral a quienes se les tomaron ambas muestras de orina para el cultivo correspondiente.

\section{Tiempo de permanencia de la sonda}

El promedio de permanencia de la sonda fue de 83 horas en las pacientes con sonda transuretral con rangos de 72 a 144 horas. En las pacientes con cistostomía fue de 73 horas con rangos de 48 a 144 horas. Creemos que el promedio de permanencia de la sonda en la cistostomía puede ser mucho más bajo pués a muchas pacientes nuestras que iniciaron micción espontánea no se les retiró la sonda hasta completar las 72 horas.

\section{Edad de las pacientes}

La distribución por edad muestra un comportamiento similar para los dos grupos (me $=56$ y 55 años) como era de esperarse ya que las pacientes escogidas al azar están dentro de los mismos rangos de edad (cuadro No. 1).

\section{Valoración del residuo urinario}

El promedio de residuo vesical en las pacientes con cistostomía fue de 22.3 cc con rangos de 5 a 200 cc. En las pacientes con sonda transuretral fue de 69.8 cc con rangos de 0 a 550 cc. Dicho residuo vesical medio difiere significativamente entre uno y otro método, en

Cuadro No. 1

RANGO Y MEDIANA DE EDAD DE LAS PACIENTES ESTUDIADAS

\begin{tabular}{|c|c|c|}
\hline PARAMETROS & TRANSURETRAL & CISTOSTOMIA \\
\hline RANGO (Límites) & $29-70$ & $36-72$ \\
\hline MEdiana & 56 & 55 \\
\hline
\end{tabular}


una relación de 1 a 3 en favor de cistostomía, y con una $\mathrm{P}<0.05$ (gráfico No. 1).

Gráfico No. 1

\section{RESIDUO MEDIO (C.C.) SEGUN METODO UTILIZADO}

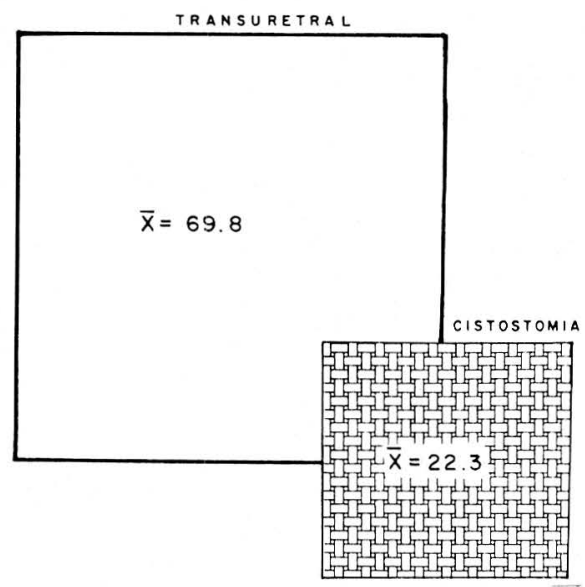

Con respecto al tiempo de permanencia de la sonda se da igualmente una relación directamente proporcional (cuadro No. 2).

Cuadro No. 2

RELACION ENTRE TIEMPO Y MEDIA (C.C.) DEL RESIDUO SEGUN METODO EMPLEADO

\begin{tabular}{|c|c|c|}
\hline $\begin{array}{c}\text { TIEMPO } \\
\text { HOrAs }\end{array}$ & $\begin{array}{c}\text { TRANSURETRAL } \\
n=30\end{array}$ & $\begin{array}{c}\text { CISTOSTOMIA } \\
n=37\end{array}$ \\
\hline 48 & 0 & 24 \\
\hline 72 & 26 & 18 \\
\hline 96 & 210 & 200 \\
\hline 120 & 173 & 0 \\
\hline 144 & 225 & 0 \\
\hline Medio general. & $69.8 *$ & 22.3 \\
\hline
\end{tabular}

$-p<0.09$
Finalmente puede observarse que la mayoría de las pacientes del método cistostomía presentan mínimos valores de residuo vesical (gráfico No. 2).

Gráfico No. 2

RESIDUO VESICAL (C.C.) SEGUN METODO EMPLEADO

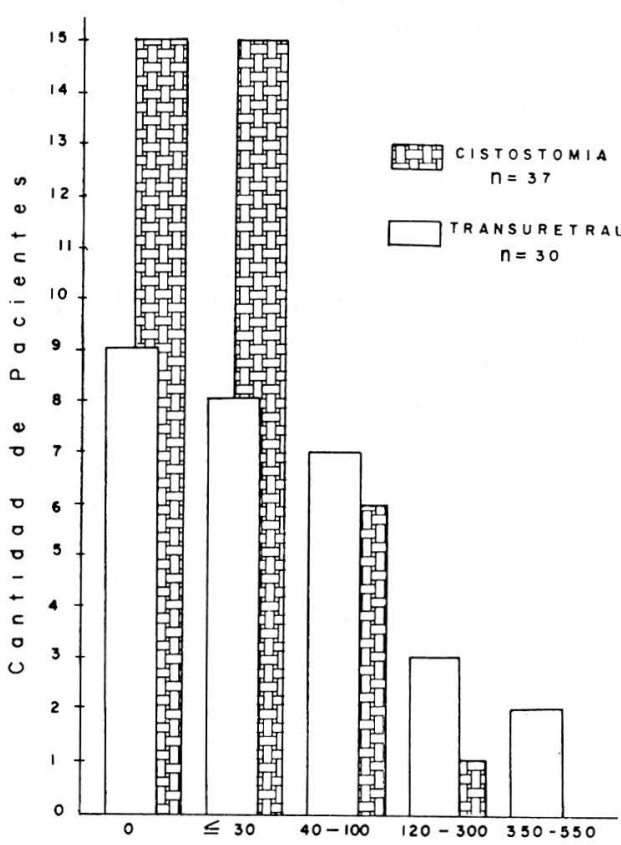

\section{Promedio de días de hospitalización}

El promedio de días de hospitalización fue sensiblemente igual en todas nuestras pacientes: cinco días y medio para las pacientes con sonda transuretral y cinco días para las pacientes con cistostomía. También debemos suponer que este promedio es menor para las pacientes con cistostomía si se tiene en cuenta que muchas pacientes que se les da de alta un día, no salen hasta el día o días siguientes porque viven lejos $y$ tienen que esperar a que los familiares vengan por ellas. 


\section{Infecciones urinarias postoperatorias}

Sobre el total de 30 pacientes con sonda transuretral completamente estudiadas obtuvimos 19 segundos cultivos urinarios positivos con más de 100.000 colonias de gérmenes, lo que nos da un promedio de $63.3 \%$ de infecciones postoperatorias. En el grupo de 37 pacientes con cistostomía tuvimos 13 muestras positivas en el segundo cultivo, o sea un $35.1 \%$. La tasa porcentual de segundos cultivos positivos difiere significativamente según el método: $P<0.05$ (gráfico No. 3).

\section{Gráfico No. 3}

TASA PORCENTUAL DE $2^{\circ}$. CULTIVOS POSITIVOS SEGUN METODO EMPLEADO

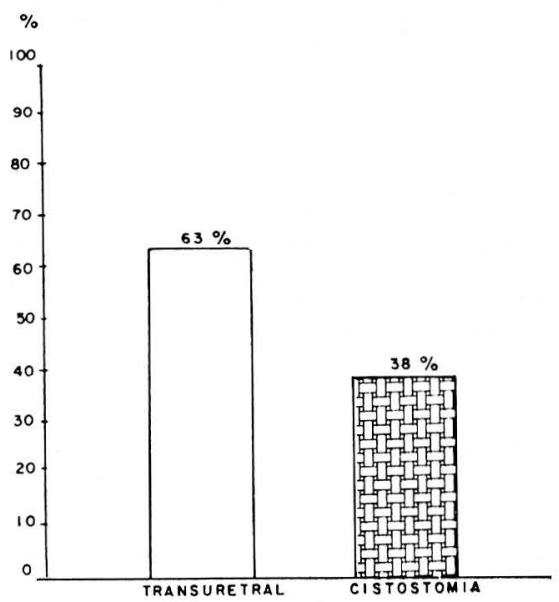

Con relación al tiempo de permanencia de la sonda los máximos porcentajes de positividad están a las 96 horas (cuadro No. 3).

Los gérmenes aislados en las 32 segundas muestras positivas fueron los siguientes en orden de frecuencia:

$\begin{array}{lc}\text { Estafilo-estreptococos } & 49 \% \\ \text { E. coli } & 37 \% \\ \text { Klebsiella } & 13 \% \\ \text { Proteus } & 1 \%\end{array}$

Al estudiar el tipo de gérmen con relación al método de drenaje vesical encontramos que no hubo diferencia con respecto al grupo de cocos pero con respecto a los enteropatógenos los encontramos dos veces más altos en las pacientes con drenaje transuretral.

Cuadro No. 3

TASA PORCENTUAL DE 2. CULTIVOS POSITIVOS SEGUN TIEMPO $Y$ METODO EMPLEADO

\begin{tabular}{|c|c|c|}
\hline $\begin{array}{c}\text { TIEMPO } \\
\text { HOROS }\end{array}$ & $\begin{array}{c}\text { TRANSURETRAL } \\
n=30\end{array}$ & $\begin{array}{c}\text { CISTOSTOMIA } \\
n=37\end{array}$ \\
\hline 48 & & $25 \%$ \\
\hline 72 & $60.8 \%$ & $45.8 \%$ \\
\hline 96 & $100 \%$ & $50 \%$ \\
\hline 120 & $67 \%$ & \\
\hline I44 & $50 \%$ & $37.8 \%$ \\
\hline TOTAL. & $63 \% *$ & \\
\hline
\end{tabular}

$* P<0.08$

Es importante anotar que solamente 14 pacientes fueron tratadas para su infección urinaria, las otras 18 pacientes salieron del Hospital asintomáticas antes de que llegaran los resultados de laboratorio.

\section{Aceptación por parte de la paciente}

Como es obvio no es posible sacar conclusiones comparativas en este rubro, pero las encuestas hechas a las pacientes dan como resultado una mejor acepta- 
ción de la cistostomía que de la sonda transuretral ya que ésta incomoda definitivamente a todas las pacientes.

\section{CONCLUSIONES}

En nuestro afán de disminuir la tasa de infecciones urinarias postoperatorias en las histerectomías vaginales en donde hay necesidad de dejar un cateter vesical para drenaje durante más de 48 horas, hicimos este estudio prospectivo comparativo utilizando como alternativa al drenaje vesical tradicional una técnica de drenaje por cistostomía fácil de realizar, usando una sonda de Folley común que una vez insertada no se sale de la vejiga, es bien tolerada y deja er libertad a la uretra para que la micción se inicie espontáneamente una vez que la vejiga recupera su función.

Las pocas complicaciones que tuvimos por dificultad en la técnica creemos son totalmente evitables en manos experimentadas $y$ de todas maneras se justificaron con la reducción en la tasa de infecciones postoperatorias casi en un $50 \%$ en éstas pacientes.

También obtuvimos una disminución muy notable en el tiempo de permanencia de la sonda vesical y en el residuo urinario, fuera de que las pacientes toleran mejor y se sienten más cómodas con una cistostomía que con la sonda a través de la uretra.

Aunque la incidencia de infecciones urinarias postoperatorias no bajó en nuestro trabajo a las cifras que dan otros autores, la diferencia entre las dos técnicas si es muy significativa y por lo tanto la recomendamos para los hospitales que en razón de su situación presupuestal no pueden disponer de equipos costosos de cistostomía.

\section{EVALUATION OF THE SUPRAPUBIC BLADDER DRAINAGE TECHNIQUE WITH CATHETER OF FOLLEY COMPARED TO THE TRANSURETRAL BLADDER DRAINAGE WITH THE SAME TYPE OF CATHETER IN THE VAGINAL HYSTERECTOMIES.}

\section{CONCLUSIONS:}

In our interest to decrease the rate of urinary infections after surgery in tive vaginal hysterectomies where is the need to leave a bladder catheter for drainage during more than 48 hours, we made a prospective comparative study using as an alternative the traditional bladder drainage a technique for drainage in cystostomy easy to realize, using the common catheter of Folley that once inserted does not come out of the bladder, it is well tolerated and it leaves the urethra free so that micturition begins spontaneously once the bladder recuperates its function.

The only complications that we had were difficulties in the technique, this can be avoided using experimented hands, anyways, this was justified with the reduction on the rate of after surgery infections almost in $50 \%$ of these pacients.

We also obtained a very notable decrease in the duration of the bladder catheter and the urinary residue, besides, the pacients tolerate better and feel a lot 
more confortable with a cystostomy than with a catheter across their urethra.

Even though the incidence of after surgery urinary infections did not decrease in our study compared to the

\section{BIBLIOGRAFIA}

1. DARMALT E., RIAÑO M., LUCENAE., OCHOA R. Cistocentesis suprapúbica en cirugía vaginal. Revista Colombiana de Obstetricia y Ginecologia Vol. XX No. 2: 125,1969

2. FRYMIRE L.J. Comparison of suprapubic versus folley drains. Obstetrics and Gynecology Vol. 38 No. 2: 239, 1971.

3. GOLDBERG B.B. and MEYER H. Ultrasonically guided suprapubic urinary bladder aspiration. Pediatrics Vol. 51 No. 1: 70. 1973

4. GUZMAN R.D. Manejo de la sonda vesical en el postoperatorio de la cirugia vaginal. Revista Colombiana de Obstetricia y Ginecología Vol. XV No. 6: 533 1964

5. HALE R.W. and Mc. CORRISTON C.C. Suprapubic cystostomy with a polyethy. lene tube. American journal of Obst and Gynecol Vol. 103: 1181. 1969.

6. HOSDKINSON P. and HODARI A. Trocar suprapubic cystostomy for postoperative bladder drainage in the female. American Journal of Obst. and Gynecol. Vol. 96: 773. 1966.

7. JACKSON G.W. Suprapubic postoperative bladder drainage. American Journal of Obst. and Gynecol. Vol. 105: 59 1969.

8. JUBIZ A., VELEZ S. Estudio sobre infección urinaria en cirugía ginecológica. Revista Colombiana de Obstetricia y Ginecologia Vol. 20 No. 2:125. 1969. numbers given by other authors, the difference between both techniques is very significative and therefore we recommend it for hospitals that because of their financial situation cannot afford expensive equipments for cystostomy.

9. Mc DUFF H.C. Suprapubic urinary drainage in Gynecologic Surgery. The Ame. rican Journal of Surgery Vol. 123: 489 1972.

10. MOODY T.E., HOWARDS S.S., SCH.. NEIDER J.A., RUDOLF L.E. Intestinal obstrucción: A complication of percu- = taneous cystostomy. A case report. The journal of urology Vol. 118: 680. 1977.

11. PELOSO O.A., WILKINSON L., FLOYD V.T. Suprapubic bladder drainage in general surgery. Arch. Surgery Vol. 106: 568. 1973.

12. REUTER H.J., LAWRENCE W.J. Physiologic low pressure irrigation for transurethral resection suprapubic trosar drainage. The journal of urology $\mathrm{Vol}$. 111: 210. 1974.

13. TAYLOR B.D., NICKEL J.E. Suprapubic cystostomy and the use of polyethylene tubing. Obstetrics and Gynecology Vol. 28 No. 6:854. 1966.

14. WHEELES CLIFFORD R. Atlas de Cirugía Pelviana. Editorial Médica Panamericana, Buenos Aires pág. 108-109, 1981.

15. SHUTE W.B., MACKINNON K.J. Postoperative restoration of micturition with suprapubic catheterization. American Journal of Obstetrics Gynecology Vol. 106: 943. 1970.

16. WILSON E.A., SPRAGUE A.D., NA. GELL J.R. Jr. Suprapubic cystostomy in Gynecologic Surgery: A comparison of two methods. American Journal of Obst. and Gynecol. 991-994. April 1 1973. 